\title{
COLLISION RISK ASSESSMENT AND OPERATIONAL EXPERIENCES FOR LEO SATELLITES AT GSOC
}

\author{
Saika Aida (1), Michael Kirschner (2)
}
(1) DLR German Space Operations Center (GSOC), Münchner Str.20, 82234 Weßling, Germany saika.aida@dlr.de
(2) DLR German Space Operations Center (GSOC), Münchner Str.20, 82234 Weßling, Germany michael.kirschner@dlr.de

\begin{abstract}
The German Space Operations Center (GSOC) is monitoring close approaches of the operational satellites against the tracked space objects. Contrary to the controlled satellites, precise orbit information is not available for a massive space objects. Currently, the TLE (TwoLine Elements) catalogue maintained by the USSTRATCOM (US Strategic Command) constitutes the only publicly available and reasonably comprehensive orbit information, which has been used for such a monitoring. In addition to TLEs, warnings from the Joint Space Operations Center (JSpOC) can be recently used as another source for the proximity prediction. Although JSpOC provides orbit information including covariance information with a relatively higher accuracy, its availability is limited and the accuracy is still not enough for a maneuver decision and also for a proper planning of an avoidance maneuver. An orbit refinement using a radar tracking is therefore foreseen in case of a critical close approach.

This paper describes the operational collision avoidance system, followed by the discussion of the orbit prediction accuracy of the TLE propagation as well as the numerical propagation from the operational point of view. The radar tracking accuracy is additionally presented for a comparison. $A$ recent close approach of TerraSAR-X is presented as an example of the event handling together with the radar tracking results performed for the debris, followed by the operational experiences for the last 1.5 years.
\end{abstract}

Keywords: Collision risk, Space debris, Orbit accuracy, Radar tracking, TLE.

\section{Introduction}

The ever increasing number of objects in the near Earth region has been causing growing concerns about the space environment and therefore about the safety of future space missions. Since most of orbital debris stay in the orbit for years, even a single collision between space objects could seriously increase the debris population, making further collision events more and more likely. The encounter of IRIDIUM 33 with COSMOS 2251 in January 2009 was the first accidental collision between two artificial satellites, which created roughly 1500 tracked debris and other small fragments still orbiting in the wide range of the LEO. In early 2010 , the close approach of $8000 \mathrm{~kg}$ ENVISAT (controlled by ESA) with a $1500 \mathrm{~kg}$ upper stage from a Chinese rocket would have lead to serious consequences, if no proper avoidance maneuver would have been performed (for details refer to [1]). These recent accidents clearly indicate the critical situation of the current debris environment as well as the importance of the operational collision avoidance.

GSOC has been implementing a collision avoidance system since 2008. The close approach monitoring is daily running in an automated process since November 2009, which detects the upcoming conjunction events of the operational four satellites in the altitude range of 460-510 km against roughly 15000 space objects listed in the TLE catalogue provided by USSTRATCOM. In addition, the warning from the Joint Space Operations Center (JSpOC) is currently an additional source for the close approach prediction, providing detail orbit information in the Conjunction Summary Message (CSM), which has become available for GSOC since July 2010. In the daily close approach monitoring, a detected collision risk is analyzed more in detail, when the pre- 
defined thresholds are violated. In case of a warning from JSpOC, the prediction is updated based on the precise orbit of the locally operated satellite together with the orbit data of the object given by JSpOC. Even after the careful risk assessment, an overly trust in the orbit information is not adequate for the decision of the maneuver planning due to its uncertainty. For this reason, orbit refinement using a radar tracking is foreseen. The influence of recent maneuvers of active satellites should not also be dismissed. In the collision avoidance operation at GSOC for nearly 1.5 years so far, the avoidance maneuvers were done for three cases for TerraSAR-X, and one for TanDEM-X, which was launched in June 2010 and flies now in a close formation with TerraSAR-X, where the minimum distance is about $450 \mathrm{~m}$.

Following the presentation of the operational collision avoidance procedure at GSOC, this paper will discuss the orbit prediction accuracy including the operational aspects. In addition to the orbit propagation accuracy both for TLE and numerical propagator, the radar tracking accuracy is also discussed based on the comparison with precise orbit data. An exemplary event handling of TerraSAR-X is furthermore addressed together with the radar tracking campaign performed for the debris, followed by the operational results of the collision avoidance for the LEO satellites in the past 1.5 years.

\section{Collision Avoidance Procedure}

As of January 2010, four operational LEO satellites are monitored in the collision avoidance system at GSOC; TerraSAR-X $(514 \mathrm{~km})$, TanDEM-X $(514 \mathrm{~km})$, and GRACE-1\&2 $(460 \mathrm{~km})$. The overview of the GSOC collision avoidance procedure is shown in Fig. 1.

The procedure comprises mainly three steps;

1. Search for potential collision risk

2. Orbit refinement by radar tracking

3. Precise collision risk assessment and planning of possible avoidance maneuver

In the first step, the potential close approach is detected and the risk assessment is performed in case of a high risk. In the daily monitoring, predicted conjunction events in the upcoming 7 days are listed in a report file, if a distance to a jeopardizing object violates the pre-defined distance thresholds; the minimum distance $<10 \mathrm{~km}$ and the radial distance $<3 \mathrm{~km}$. These thresholds were derived from the TLE accuracy analysis described in [3]. The prediction is updated twice a day automatically, and reports are sent to the FD staff. The latest prediction report is also available on the internal flight dynamics website, so that the GSOC staff can share the information about the upcoming close approach. When a collision probability exceeds the current threshold of $10^{-4}$, the criticality of the event is closely analyzed. In addition to the daily TLE-based prediction, the warning message (CSM) from JSpOC has been another source for detection of the critical close approach since mid of 2010. In case of LEO satellites, the notification by the CSM is currently provided, when the minimum distance is $<1 \mathrm{~km}$, the radial distance $<200 \mathrm{~m}$ and the time to the closest approach $<72$ hours as described in [2]. When a CSM is received, the prediction is updated based on the latest orbit data of the operational satellite as well as those of the jeopardizing object derived from the CSM.

The orbit refinement of the jeopardizing object using a radar tracking is planned as the second step, if a high collision is expected from the risk assessment in the previous step. The accuracy of such a radar tracking was investigated in [3], showing enormous reduction of the orbit uncertainty compared to TLEs. Even for JSpOC warnings, which provide a relatively high orbit accuracy, the given covariance is often too large for a proper decision of taking a maneuver especially for small objects. Therefore, a radar tracking is performed if available to get the latest and the more precise orbit of the object. 
At the final step, the prediction is updated based on the latest information. The criticality of the conjunction is assessed again in terms of the collision probability as well as the proximity geometry, and a collision avoidance maneuver is planned when necessary. The maneuver decision is made 0.5-1.0 day prior to the Time of the Closest Approach (TCA).

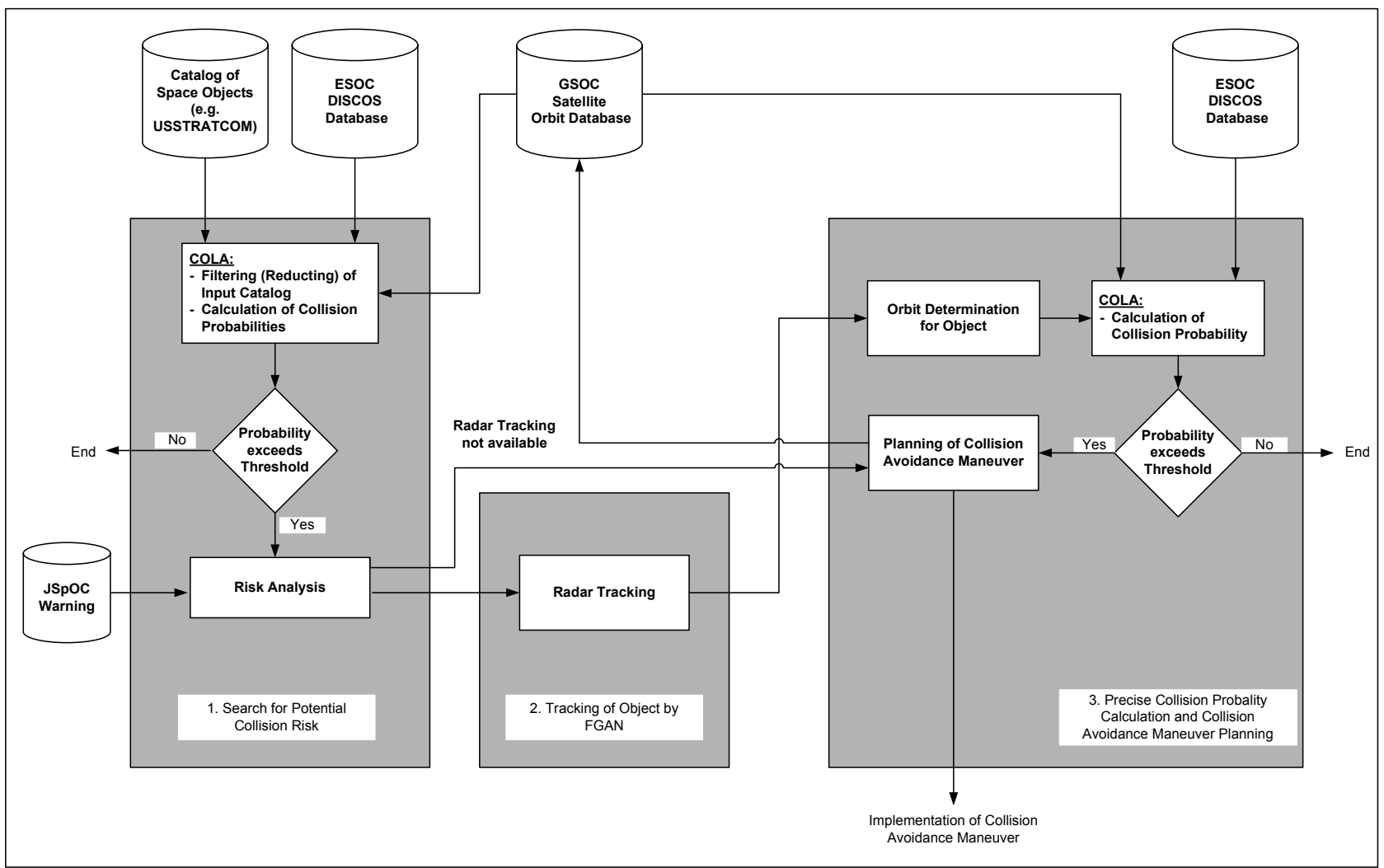

Figure 1. Collision Avoidance Procedure

\section{Orbit Prediction Accuracy Analysis}

In order to derive criteria for critical conjunctions, an analysis for the OP (Orbit Prediction) accuracy was performed. Propagation errors of ephemerides generated from USSTRATCOM TLEs and those generated by numerical orbit propagation were investigated by comparing the orbits with the precise orbits of locally operated satellites CHAMP and GRACE-1 (at an altitude of 270$430 \mathrm{~km}$, and 460-500 km respectively). By using the long period of data, the dependency of the prediction accuracy on the altitude as well as the solar flux was obtained as shown in [4]. This analysis was extended for TerraSAR-X to get the prediction error at a higher altitude of $510 \mathrm{~km}$. The resulting RMS can be operationally used to generate the covariance of space objects in the relevant altitude and solar flux range. In addition, the numerical propagation errors can be also applied as a covariance information of the locally operated satellites instead of propagating an initial covariance matrix, which does not include the influence of the solar flux prediction error and could results in a too optimistic estimation of orbit uncertainties.

The well established OD (Orbit Determination) and OP software ODEM (Orbit Determination for Extended Maneuvers) was used to generate ephemerides based on numerical propagation. The OD inside ODEM is formulated as a sequential non-linear least-squares problem based on Givens rotations and the OP is based on a standard numerical integration method for initial value problems. In particular an Adams-Bashforth-Moulton method for numerical integration of ordinary differential equations is adopted. This method employs variable order and step-size and is particularly suited for tasks like the prediction of satellite orbits. The numerical orbit propagator is 
using a comprehensive model for the acceleration of an Earth orbiting spacecraft under the influence of gravitational and non-gravitational forces.

The 'real orbit' as reference was generated by the software modules POSFIT or RDOD, which are part of the GHOST (GPS High Precision Orbit Determination Software Tool) package developed by GSOC/DLR. POSFIT performs a reduced dynamic orbit determination from a given a priori orbit. It estimates initial conditions, dynamical model parameters and empirical accelerations in a least squares fit. In addition, RDOD uses raw GPS measurements as observations for a precise orbit determination (POD). The position accuracy of the orbits based on POSFIT and POD is better than $2 \mathrm{~m}$ and $10 \mathrm{~cm}$, respectively.

\subsection{TLE Orbit Propagation Accuracy}

Errors of the propagated TLEs w.r.t. POD orbits in the LEO region were investigated for a period of low solar flux in [3]. On the other hand, the analysis of the TLE fit against osculating orbit ephemerides showed that the solar activity can have an important influence on the prediction error. As the solar activity is slowly increasing since end of 2009, it is also important to know more in detail the influence of the solar activity on the orbit prediction. Therefore the orbit prediction accuracy analysis was extended to investigate the dependency of the prediction accuracy not only on the altitude but also on the solar flux, using orbit data of a long period. For two satellite missions at GSOC, CHAMP and GRACE, GPS orbits are available during the whole bandwidth of the solar activity, since CHAMP was launched in 2000 and GRACE in 2002.

In the analysis, TLEs for each satellite were propagated to the corresponding POD epoch up to 7 days (forwards) using the SGP4 propagator. The resulting orbits were compared with the precise orbits of CHAMP (April 2001-July 2010), GRACE-1 (April 2002-July 2010) and also TerraSAR-X (July 2007-July 2010) which are available at an interval of 30 seconds.

Table 1. RTN Error of TLE Propagation (RMS in [m])

\begin{tabular}{|c|c|c|c|c|c|c|c|c|c|c|c|c|c|c|c|c|}
\hline & & \multicolumn{5}{|c|}{1 day prop } & \multicolumn{5}{|c|}{4 days prop } & \multicolumn{5}{|c|}{7 days prop } \\
\hline & & & & & $u x$ & & & & $F l$ & $u x$ & & & & & $u x$ & \\
\hline \multirow{7}{*}{$\mathrm{R}$} & & & -90 & $90-140$ & 140-190 & $190-$ & & -90 & $90-140$ & 140-190 & 190- & & -90 & $90-140$ & 140-190 & 190- \\
\hline & & -300 & 309 & 208 & 275 & 225 & -300 & 748 & 622 & 682 & 636 & -300 & 2693 & 2498 & 3227 & 3446 \\
\hline & ह & $300-350$ & 353 & 252 & 320 & 269 & $300-350$ & 667 & 541 & 601 & 555 & $300-350$ & 1007 & 812 & 1541 & 1761 \\
\hline &  & $350-400$ & 285 & 234 & 301 & 251 & $350-400$ & 579 & 507 & 566 & 520 & $350-400$ & 988 & 955 & 1684 & 1904 \\
\hline & $\stackrel{\Xi}{2}$ & $400-450$ & 351 & 305 & 214 & 163 & $400-450$ & 559 & 491 & 451 & 405 & $400-450$ & 835 & 780 & 764 & 983 \\
\hline & $\stackrel{\Xi}{\approx}$ & $450-500$ & 333 & 293 & 369 & 317 & $450-500$ & 622 & 558 & 628 & 591 & $450-500$ & 912 & 836 & 953 & 884 \\
\hline & & $500-$ & 219 & 178 & 254 & 203 & $500-$ & 330 & 267 & 337 & 299 & $500-$ & 466 & 389 & 506 & 438 \\
\hline \multirow{7}{*}{$\mathrm{T}$} & & & -90 & $90-140$ & 140-190 & 190 & & -90 & $90-140$ & 140-190 & 190- & & -90 & $90-140$ & $140-190$ & 190- \\
\hline & & -300 & 2925 & 1721 & 2035 & 2256 & -300 & 40669 & 45697 & 50981 & 57079 & -300 & 168530 & 136560 & 168530 & 189108 \\
\hline & ह & $300-350$ & 2963 & 1758 & 2073 & 2294 & $300-350$ & 12159 & 17187 & 22471 & 28568 & $300-350$ & 35184 & 38817 & 70787 & 91365 \\
\hline & 0 & $350-400$ & 1528 & 1564 & 1878 & 2099 & $350-400$ & 14163 & 15652 & 20936 & 27034 & $350-400$ & 45122 & 47606 & 79577 & 100154 \\
\hline &. & $400-450$ & 1396 & 1438 & 1348 & 1569 & $400-450$ & 8572 & 10802 & 12476 & 18574 & $400-450$ & 30609 & 36578 & 37855 & 58432 \\
\hline & $\stackrel{\vec{I}}{=}$ & $450-500$ & 1312 & 1358 & 2343 & 2476 & $450-500$ & 3830 & 6799 & 10747 & 18224 & $450-500$ & 8240 & 17694 & 34904 & 43721 \\
\hline & & $500-$ & 1207 & 1254 & 2239 & 2372 & $500-$ & 2519 & 5489 & 9436 & 16913 & $500-$ & 5456 & 14910 & 32120 & 40937 \\
\hline \multirow{7}{*}{$\mathrm{N}$} & & & -90 & $90-140$ & 140-190 & $190-$ & & -90 & $90-140$ & $140-190$ & 190- & & -90 & $90-140$ & $140-190$ & 190- \\
\hline & & -300 & 482 & 409 & 495 & 502 & -300 & 575 & 477 & 514 & 530 & -300 & 669 & 453 & 451 & 481 \\
\hline & है & $300-350$ & 375 & 302 & 388 & 395 & $300-350$ & 431 & 334 & 370 & 386 & $300-350$ & 490 & 274 & 272 & 302 \\
\hline & 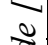 & $350-400$ & 248 & 300 & 386 & 393 & $350-400$ & 256 & 303 & 340 & 356 & $350-400$ & 283 & 320 & 318 & 348 \\
\hline & $\stackrel{2}{2}$ & $400-450$ & 338 & 347 & 375 & 383 & $400-450$ & 334 & 323 & 359 & 375 & $400-450$ & 338 & 310 & 357 & 387 \\
\hline & $\stackrel{\Xi}{=}$ & $450-500$ & 324 & 290 & 401 & 367 & $450-500$ & 358 & 289 & 393 & 368 & $450-500$ & 402 & 309 & 390 & 401 \\
\hline & & $500-$ & 418 & 383 & 494 & 461 & $500-$ & 461 & 392 & 496 & 470 & $500-$ & 499 & 406 & 486 & 497 \\
\hline
\end{tabular}


RMS errors sorted by the altitude and the solar flux at each POD epoch are shown in Tab. 1. Since not enough data were available to cover all the altitude-flux sets, some RMS errors were substituted with the estimated value using linear extrapolation just to see the tendency of the error growth at the wider range of the altitude-flux set. The missing data was estimated from at least 3 surrounding cells in a $2 \times 2$ square data set, using the value at the intersection point of the two diagonals. When more than one square data set exists, the average from each square data was taken. This process was continued until all possible data are filled. In Tab. 1, such extrapolated data are distinguished from the statistical results by the dark pattern.

As a whole, the RMS errors of the along-track and radial components become larger at the lower altitude and also at the higher solar flux period and grow exponentially for the longer prediction time. On the other hand, fluctuation of the solar flux is much larger during the higher flux period compared to the lower one. Due to this behavior and since the solar flux has a severe influence on the atmospheric density, the along track and also radial prediction errors are expected to become larger when the solar flux is higher and also when the altitude is lower. As for the RMS error of the cross-track component, there is no distinct dependency on the solar flux and the altitude, but the error grows gradually with the propagation length.

\subsection{Numerical Orbit Propagation Accuracy}

As done in the TLE analysis, the orbit prediction error was analyzed as well for the numerical propagation using the orbit database of CHAMP, GRACE-1 and TerraSAR-X. The orbits were propagated up to 7 days with the ODEM tool, and compared with the same precise orbits. For the numerical propagation, the predicted solar flux at the epoch of the database was used.

Table 2. RTN Error of NUM Propagation (RMS in [m])

\begin{tabular}{|c|c|c|c|c|c|c|c|c|c|c|c|c|c|c|c|c|}
\hline & & \multicolumn{5}{|c|}{1 day prop } & \multicolumn{5}{|c|}{4 days prop } & \multicolumn{5}{|c|}{7 days prop } \\
\hline & & & \multicolumn{4}{|c|}{ Flux } & & \multicolumn{4}{|c|}{ Flux } & & \multicolumn{4}{|c|}{ Flux } \\
\hline \multirow{7}{*}{$\mathrm{R}$} & & & -90 & $90-140$ & |140-190 & 190 & & -90 & $90-140$ & 140-190 & $190-$ & & -90 & $90-140$ & 140-190 & $190-$ \\
\hline & & -300 & 19 & 22 & 23 & 25 & -300 & 223 & 248 & 238 & 263 & -300 & 1505 & 1681 & 3514 & 3430 \\
\hline & है & $300-350$ & 5 & 7 & 9 & 11 & $300-350$ & 36 & 62 & 51 & 77 & $300-350$ & 125 & 301 & 2133 & 2050 \\
\hline & 2 & $350-400$ & 8 & 10 & 12 & 14 & $350-400$ & 43 & 69 & 58 & 84 & $350-400$ & 282 & 458 & 2290 & 2207 \\
\hline & 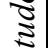 & $400-450$ & 6 & 7 & 8 & 10 & $400-450$ & 24 & 40 & 46 & 71 & $400-450$ & 67 & 170 & 204 & 120 \\
\hline & $\stackrel{\Xi}{=}$ & $450-500$ & 5 & 6 & 6 & 8 & $450-500$ & 12 & 19 & 25 & 50 & $450-500$ & 22 & 52 & 210 & 127 \\
\hline & & $500-$ & 1 & 2 & 3 & 5 & $500-$ & 8 & 14 & 20 & 46 & $500-$ & 3 & 34 & 192 & 108 \\
\hline \multirow{7}{*}{$\mathrm{T}$} & & & -90 & $90-140$ & 140-190 & $190-$ & & -90 & $90-140$ & 140-190 & $190-$ & & -90 & $90-140$ & 140-190 & 190- \\
\hline & & -300 & 1204 & 1390 & 1449 & 1584 & -300 & 28388 & 30919 & 28833 & 31719 & -300 & 97621 & 103106 & 134988 & 139301 \\
\hline & E & $300-350$ & 278 & 463 & 522 & 657 & $300-350$ & 7244 & 9775 & 7689 & 10575 & $300-350$ & 24695 & 30179 & 62062 & 66375 \\
\hline & 2 & $350-400$ & 530 & 668 & 727 & 862 & $350-400$ & 10199 & 12730 & 10643 & 13529 & $350-400$ & 34994 & 40479 & 72361 & 76674 \\
\hline & $\mathbb{2}$ & $400-450$ & 337 & 466 & 449 & 585 & $400-450$ & 6747 & 9085 & 9697 & 12582 & $400-450$ & 22520 & 28769 & 36506 & 40819 \\
\hline & $\stackrel{\vec{I}}{\vec{\nabla}}$ & $450-500$ & 97 & 217 & 332 & 468 & $450-500$ & 1652 & 3796 & 5988 & 8874 & $450-500$ & 5382 & 12395 & 22299 & 26612 \\
\hline & & $500-$ & 22 & 142 & 257 & 392 & $500-$ & 376 & 2520 & 4712 & 7598 & $500-$ & 951 & 7964 & 17868 & 22181 \\
\hline \multirow{7}{*}{$\mathrm{N}$} & & & -90 & $90-140$ & 140-190 & $190-$ & & -90 & $90-140$ & $140-190$ & 190- & & -90 & $90-140$ & $140-190$ & 190- \\
\hline & & -300 & 3 & 3 & 3 & 4 & -300 & 10 & 10 & 10 & 11 & -300 & 19 & 19 & 21 & 20 \\
\hline & $\underline{\Sigma}$ & $300-350$ & 2 & 2 & 2 & 2 & $300-350$ & 5 & 5 & 5 & 6 & $300-350$ & 8 & 9 & 11 & 10 \\
\hline & $\stackrel{0}{\sim}$ & $350-400$ & 1 & 1 & 1 & 1 & $350-400$ & 4 & 4 & 5 & 5 & $350-400$ & 7 & 8 & 10 & 9 \\
\hline & $\stackrel{2}{2}$ & $400-450$ & 1 & 1 & 1 & 2 & $400-450$ & 4 & 4 & 5 & 6 & $400-450$ & 7 & 8 & 10 & 9 \\
\hline & $\stackrel{\Xi}{\Xi}$ & $450-500$ & 6 & 6 & 5 & 6 & $450-500$ & 14 & 14 & 10 & 11 & $450-500$ & 23 & 24 & 17 & 16 \\
\hline & & $500-$ & 1 & 1 & 1 & 1 & $500-$ & 4 & 4 & 1 & 1 & $500-$ & 5 & 6 & 0 & 0 \\
\hline
\end{tabular}

The resulting RMS errors in Tab. 2 show again the dominant prediction error in the along-track direction. Comparable to the TLE analysis, the along-track and radial errors become larger at the lower altitude and at the higher solar flux period. The RMS error of the along-track component does not show the clear dependency on the solar flux and the altitude, but the error grows gradually with 
the longer propagation. By propagating orbits using the well-modeled propagator, errors are small especially for the radial and along-track components and also for the along-track component during the short-term propagation. However, the longer propagation results in a bad orbit prediction especially in the along track direction. The reason could be a prediction error of the solar flux, which becomes larger at the higher solar flux period.

Table 3 shows the difference (in the mean and the standard deviation) between the predicted and the real solar flux values for different prediction periods. Flux data of the last 10 years (January 2001July 2010) was taken for the analysis, where for each day a dedicated flux file was used containing 8-day-prediction data available at that day. These flux files are based on archived daily short-term predictions by ESOC. The results clearly show the growing prediction error for higher solar flux values, leading to the large along-track and radial error in the numerical propagation, even with the well-established model of the propagator.

Table 3. Solar Flux Prediction Error (in $10^{-22}\left[\mathrm{Ws} / \mathrm{m}^{2}\right]$ )

\begin{tabular}{|c|c|c|c|c|c|c|c|}
\hline & & \multicolumn{2}{|c|}{1 day pred. } & \multicolumn{2}{c|}{2 day pred } & \multicolumn{2}{c|}{3 day pred } \\
\hline & & Mean & $1 \sigma$ & Mean & $1 \sigma$ & Mean & $1 \sigma$ \\
\hline \multirow{4}{*}{} & -90 & 0.5 & 3.0 & 1.3 & 4.9 & 1.8 & 5.9 \\
\cline { 2 - 8 } & $90-140$ & 1.4 & 11.6 & 3.5 & 16.8 & 5.3 & 19.2 \\
\cline { 2 - 8 } & $140-190$ & 1.3 & 12.6 & 1.4 & 20.6 & 2.6 & 25.3 \\
\cline { 2 - 8 } & $190-$ & -6.4 & 21.7 & -15.4 & 33.3 & -23.6 & 39.1 \\
\hline
\end{tabular}

In the operational close approach prediction, the obtained RMS errors are implemented as a covariance information of the numerical orbit propagation for the operational satellites, for which the precise orbits are known. On the other hand, this analysis showed that the numerical propagation can result in a large orbit error for the long time prediction, although it is still better than the TLE propagation. However, the orbit prediction in the radial and along-track direction for the numerical propagation is very precise for the shorter period of the prediction around 2-3 days, and even better around 1-1.5 days, which is the decision point for the radar tracking and the maneuver planning respectively.

\subsection{Radar Tracking Accuracy}

In case of a critical conjunction, orbit refinement of the jeopardizing object using a radar tracking is foreseen. A test campaign was performed for the operational satellites TerraSAR-X $(514 \mathrm{~km})$ and CHAMP $(330 \mathrm{~km})$ and the resulting accuracy was compared with the GPS navigation solution data, as presented in [3]. For the radar tracking, the Tracking and Imaging Radar (TIRA) system of FHR was used. This is the only radar in Germany, capable to observe non-cooperative objects in space. The pattern of ground contacts is typical for near polar orbits and ground station locations like the FHR one, where up to three subsequent orbits with visibility are followed by at least 9 hours with no visibility.

The main outcome of this campaign was that the quality to the same order as the GPS navigation solution was achieved by the radar tracking. The orbit accuracy after 1.0 day orbit propagation is shown for TerraSAR-X in Tab. 4. A maximum of five passes covering 24 hours were used for the orbit determination based on the radar tracking data. Compared with the corresponding TLE propagation errors (1-day prop., Altitude $>500 \mathrm{~km}$ and Flux $<90$ in Tab. 1), the orbit accuracy could be clearly improved, which leads to a reduction of collision avoidance maneuvers as well as to a proper planning of a maneuver. 
In the operational collision avoidance strategy, a radar tracking has to be performed timely prior to the TCA. While a later timing reduces the time for the maneuver planning, an earlier one increases the prediction length, leading to a less orbit accuracy. For this reason, a radar tracking is supposed to be completed 0.5-1.0 day before the TCA. In total, four passes are planned for one tracking campaign to obtain the minimum data arc of 12 hours for the orbit determination, which is necessary due to the estimation of the ballistic coefficient under the significant influence of the atmosphere in the LEO region.

Table 4. RMS after 1.0 day TerraSAR-X Orbit Propagation (in [m])

\begin{tabular}{|l|c|c|c|c|c|c|}
\hline & \multicolumn{3}{|c|}{$32 \times 32$ gravity field } & \multicolumn{3}{c|}{$70 \times 70$ gravity field } \\
\hline & Radial & Along-track & Normal & Radial & Along-track & Normal \\
\hline FHR (12h OD) & 4 & 83 & 5 & 1 & 40 & 1 \\
\hline FHR (24h OD) & 6 & 181 & 7 & 2 & 101 & 2 \\
\hline GPS (12h OD) & 4 & 45 & 6 & 1 & 49 & 1 \\
\hline GPS (24h OD) & 4 & 83 & 5 & 1 & 61 & 1 \\
\hline
\end{tabular}

\section{Collision Avoidance Maneuver Strategy}

In case an avoidance maneuver is planned, either of the following strategies is normally considered: a change of the execution epoch or the size of an upcoming regular maneuver, or the implementation of a collision avoidance maneuver to reduce the collision probability. The former is more preferable with regard to fuel consumption and operational aspects, but its availability depends on the timing of the existing maneuver. If any change of the regular maneuver is not possible, the latter strategy is applied to increase the relative distance mostly in the radial direction, considering the mission constraints of the satellite. A Change of the radial distance is most chosen, because a separation is achieved in a shorter period and with a smaller maneuver compared to the out-of-plane direction. Additionally, orbit prediction is generally more accurate in the radial direction as shown in chapter 3. After a collision avoidance maneuver, another maneuver is often required to come back to the nominal orbit like TerraSAR-X and TanDEM-X, which are controlled against a reference orbit inside a control tube of $500 \mathrm{~m}$ diameter. These satellites are flying in a close formation with the relative distance of $<500 \mathrm{~m}$.

When a significant risk (i.e. $10^{-4}$ ) remains for TerraSAR-X (TSX) or TanDEM-X (TDX), the following precautions exist in principle (for details refer to [6]). If the risk applies only to TSX, there are three collision avoidance scenarios:

A. Change execution time and size of a regular TSX maneuver to take place before (or after) the event, TDX replicates the maneuver as usual, or

B. TSX performs two maneuvers: collision avoidance and re-acquisition of reference orbit, and

B.1 TDX replicates the maneuvers (fuel-expensive), or

B.2 TDX remains passive and the formation has to be re-acquired afterwards (timeconsuming).

Of course the risk assessment is to be repeated for every maneuver planned for TSX and/or TDX before command upload. If solely TDX is affected, TSX remains passive and TDX has to perform maneuvers for collision avoidance and formation re-acquisition. 


\section{Event handling}

The recent close approach of TerraSAR-X to a Pegasus debris is discussed in this chapter, which lead to a collision avoidance maneuver. In addition to an alert from the daily monitoring, a warning from JSpOC was also received and a radar tracking of the debris could be performed. The summary of this event is shown in Tab. 5.

Table 5. Summary of Events

\begin{tabular}{|l|c|l|}
\hline Object & & Pegasus debris (ID 24978) \\
\hline Estimated size & & $\sim 10 \mathrm{~cm}\left(\mathrm{RCS}: 0.010 \mathrm{~m}^{2}\right)$ \\
\hline Eccentricity, Inclination & & $e: 0.044, i: 81.5^{\circ}$ \\
\hline Perigee/Apogee altitude & {$[\mathrm{km}]$} & $464 / 1181$ \\
\hline TCA & {$[\mathrm{UTC}]$} & $2010 / 08 / 07 \quad 13: 19: 35$ \\
\hline Relative velocity & {$[\mathrm{km} / \mathrm{s}]$} & 15.1 \\
\hline Orbital plane angle & {$[\mathrm{deg}]$} & 160 \\
\hline
\end{tabular}

\subsection{Close Approach of TerraSAR-X}

The event had been constantly predicted since the earliest prediction of 7 days before the TCA, with the maximum probability of $\sim 10^{-4}$ (Tab. 6-A). On the other hand, the flight dynamics staff received a warning from JSpOC $\sim 1.5$ days before the TCA, with a minimum distance of $90 \mathrm{~m}$ (Tab. 6-B). The event was analyzed using the precise orbit of TerraSAR-X (accuracy of a few meters in the radial direction as shown in Tab. 2, for a 1-day prop., altitude $>500 \mathrm{~km}$ and flux $<90$ ), together with the orbit information of the debris provided by JSpOC (Tab. 6-C). On the other hand, the orbit information of TerraSAR-X from JSpOC is based on independent radar tracking data and with the given $1 \sigma$-errors ( $3 \mathrm{~m}$ in radial, $151 \mathrm{~m}$ in along-track, and $2 \mathrm{~m}$ in cross-track). As these small $1 \sigma$ errors show, the GSOC analysis (C) is comparable with the JSpOC prediction (B). After the analysis, an orbit refinement using the TIRA system was decided to get a better orbit information of the debris. The radar tracking was planned covering four passes 1-2 days before the TCA, among which the last two passes could be used for the orbit determination. The precise orbit of the debris was determined using the resulting tracking arc of 10 hours and the close approach prediction was updated (Tab. 6-D).

Table 6. Close Approach Prediction

\begin{tabular}{|l|c|c|c|c|}
\hline & $\begin{array}{c}\text { A: Daily prediction } \\
\text { (TCA-1d) }\end{array}$ & $\begin{array}{c}\text { B: JSpOC warning } \\
\text { (TCA-1.5d) }\end{array}$ & $\begin{array}{c}\text { C: GSOC analysis } \\
\text { (TCA-1.5d) }\end{array}$ & $\begin{array}{c}\text { D: FHR tracking } \\
\text { (TCA-1d) }\end{array}$ \\
\hline TX1 orbit & TX1 precise & JSpOC & TX1 precise & TX1 precise \\
\hline Debris orbit & TLE (TCA-2.7d) & JSpOC & JSpOC & Radar tracking \\
\hline Probability & $1.39 \mathrm{E}-05$ & N/A & $3.44 \mathrm{E}-04$ & $1.13 \mathrm{E}-03$ \\
\hline Min.distance & 1.064 & 0.090 & 0.081 & 0.216 \\
\hline $\mathrm{R}$ (Radial) & 0.166 & 0.069 & 0.071 & -0.019 \\
\hline T (Along-track) & -0.184 & -0.007 & 0.005 & -0.031 \\
\hline $\mathrm{N}$ (Cross-track) & -1.035 & -0.058 & 0.039 & -0.213 \\
\hline Orbital arc dist. & 0.197 & N/A & 0.070 & 0.013 \\
\hline
\end{tabular}

Even though the latest and the most precise prediction at the time showed a larger relative distance compared with the prediction before, the close radial distance of $19 \mathrm{~m}$ and the orbital arc distance of $13 \mathrm{~m}$, which is the possible minimum distance of two orbital arcs of TerraSAR-X and the debris, were considered as critical. Therefore a collision avoidance maneuver was finally decided. Two maneuvers were performed half an orbit before and after the TCA to separate the radial distance by 
$\sim 150 \mathrm{~m}$, and then to come back to the nominal orbit. The final prediction after the maneuver planning is shown in Tab. 7. Both of the along-track and out-of-plane components were also enlarged consequently.

Table 7. Prediction incl. Avoidance Maneuver

\begin{tabular}{|l|c|}
\hline & $\begin{array}{c}\text { E: After maneuver planning } \\
\text { (TCA-0.8d) }\end{array}$ \\
\hline TX1 orbit & TX1 precise \\
\hline Debris orbit & Radar tracking \\
\hline Probability & $1.77 \mathrm{E}-05$ \\
\hline Min.distance & 0.337 \\
\hline R (Radial) & -0.165 \\
\hline T (Along-track) & -0.046 \\
\hline N (Cross-track) & -0.291 \\
\hline Orbital arc dist. & 0.174 \\
\hline
\end{tabular}

\subsection{Radar Tracking Accuracy of Debris}

For the operational orbit refinement using a radar tracking, four passes are currently planned to be necessary for the determination of an orbit using a 12-24 hours data arc. The minimum length of the 12 hours arc is required for the estimation of the ballistic coefficient under the influence of the atmosphere.

In the radar tracking campaign performed for the Pegasus debris, the tracking data from the second half of a pass (TCA-1.5 days, 2010/08/06 05:00 UTC) and a whole pass (TCA-1.0 day, 2010/08/06 15:30 UTC) could be used. The orbit of the debris was determined using the 10 hours data arc of these 1.5 passes with the estimated RMS as shown in Tab. 8. The RMS at the TCA was obtained by numerically propagating the initial value over a 1-day period from the epoch of the last measurement. Even with this short data arc, the orbit accuracy in the radial and the along-track direction could be improved compared to those given by JSpOC. Compared with the reference TLE accuracy at the corresponding column in Tab. 1 (1-day prop., Altitude $>500 \mathrm{~km}$ and Flux $<90$ ), the accuracy improvement is enormous, although other parameters such as eccentricity and inclination (for details refer to [5]) and also the object size have to be considered for more details.

The reason for the relatively poor accuracy in the out-of plane component can be explained by the positional constraints of the used passes. Figure 2 shows the azimuth (angular direction) and the elevation (radial direction) of the passes in the polar coordinate frame. The point of origin represents 90 degrees of the elevation. Both lines pass near the origin, reaching the maximum elevation of 89 degrees and 84 degrees for each pass. It means that the observation plane is almost identical to the orbital plane of the debris. Accordingly, lack of tracking information in the out-ofplane direction lead to the reduction of the orbit determination accuracy.

Compared with the test campaign results of the TerraSAR-X radar tracking as shown in Tab. 4, the RMS accuracy of the debris is comparable, even with its relatively small size $(\sim 10 \mathrm{~cm})$ and eccentric orbit. However, it has also to be mentioned that the RMS of the radar tracking in Tab. 8 was calculated by numerically propagating the initial error, whereas the results in Tab. 4 was obtained by comparing the orbits with the precise orbit data of TerraSAR-X. 
Table 8. RMS of the Pegasus Debris at TCA (in [m])

\begin{tabular}{|l|c|c|c|}
\hline & Radial & Along-track & Normal \\
\hline Radar tracking & 2.5 & 16.3 & 29.4 \\
\hline JSpOC & 12 & 135 & 18 \\
\hline
\end{tabular}

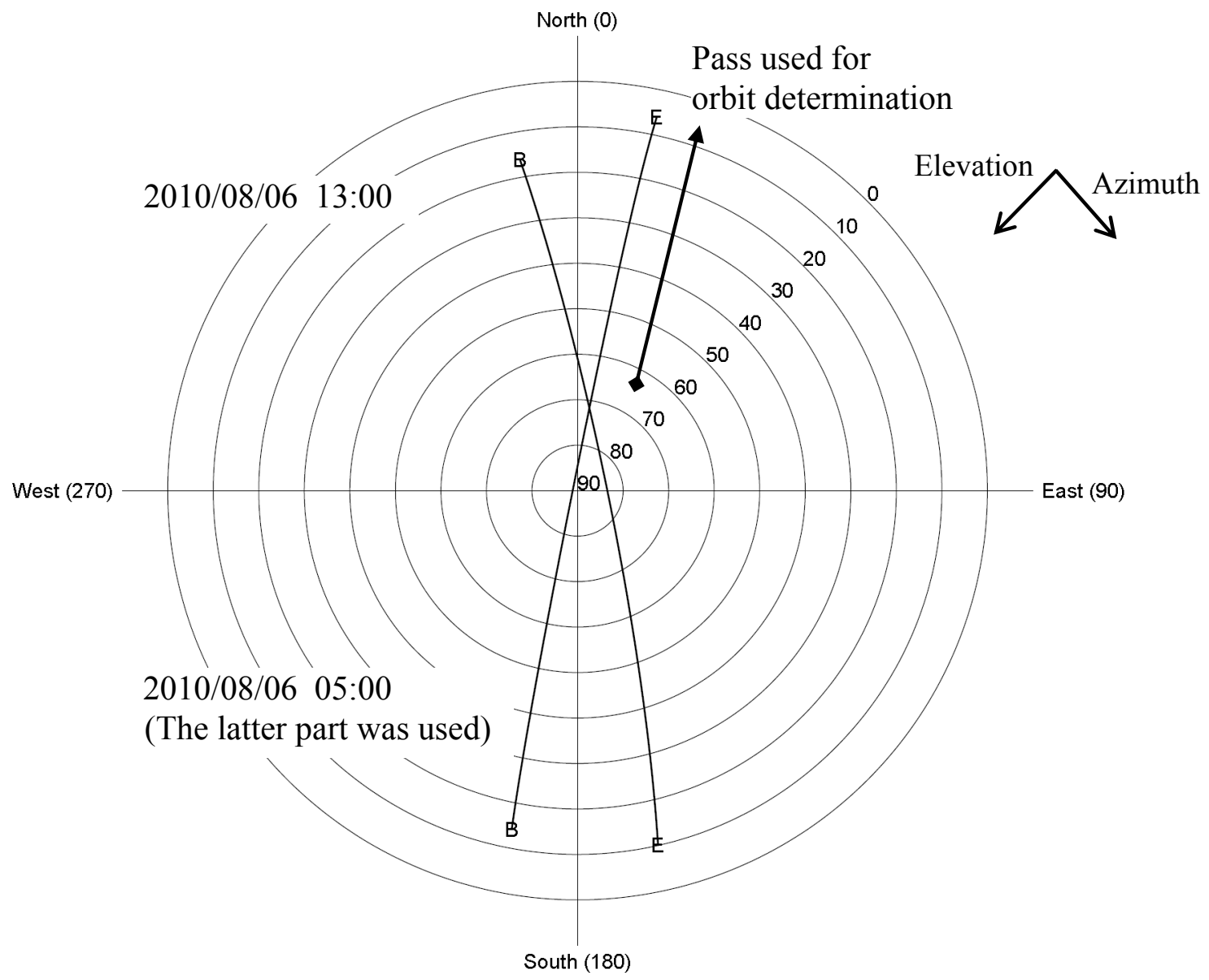

Figure 2. Radar Tracking Pass (in degree)

\section{Operational Results}

Close approaches of the operational LEO satellites TerraSAR-X, TanDEM-X (since June 2010), GRACE-1\&2, and CHAMP (until September 2010) have been monitored and handled for nearly 1.5 years since 2009 . Some cases were alarmed from daily monitoring results or from warnings by JSpOC, resulting in a further risk assessment. The past analyzed events as of January 2011 are listed in Tab. 9.

In total, three cases lead to an avoidance maneuver for TerraSAR-X, and one for TanDEM-X. These two satellites are kept in a very close formation with a relative distance of $<500 \mathrm{~m}$. No collision avoidance maneuvers have been planned for them since the close formation was established in December 2010, but the risk analyses were already performed against two space debris. In most of the cases, the analysis has to be done for both two satellites, when one of them gets an alarm. It has also to be mentioned that two close approaches for TanDEM-X on 2010/06/27 were handled by one avoidance maneuver during its LEOP, based only on TLE information. The maneuvers were executed as part of the re-planned maneuver sequence for the eccentricity correction necessary to bring the satellite into the formation with TerraSAR-X. Therefore no fuel in addition to the nominal target orbit acquisition budget was necessary. The number of the events is 
estimated to increase in the next years, as the following satellite missions such as PRISMA are operated in a higher altitude of $750 \mathrm{~km}$, which is one of the most populated regions in the space.

The relative distance and its radial component before the maneuver decision are also listed, together with the value provided by JSpOC. The jeopardizing objects are shown as well as their estimated size calculated from the radar cross section. Most are derived from debris; 4 out of 15 are Cosmos 2251 debris and 2 are Fengyun 1C debris. As shown in Tab. 10, the estimated object size is mostly smaller than $1 \mathrm{~m}$, often even smaller than $10 \mathrm{~cm}$, causing the large error in the orbit prediction. Even for large objects, given orbit accuracies of active satellites could be worse due to maneuvers. Since such orbit errors grow according to the prediction length, orbit refinement using a radar tracking is an effective way to get a more accurate orbit information. A contact to a control center can also be an alternative for the orbit refinement, if a controlled satellite is approaching.

Table 9. Analyzed Close Approaches

\begin{tabular}{|c|c|l|c|c|c|c|c|c|}
\hline $\begin{array}{c}\text { TCA } \\
{[\mathrm{UTC}]}\end{array}$ & Sat. & \multicolumn{1}{|c|}{ Object } & $\begin{array}{c}\text { Size } \\
{[\mathrm{m}]}\end{array}$ & $\begin{array}{c}\text { Dist. } \\
{[\mathrm{m}]}\end{array}$ & $\begin{array}{c}\text { R.Dist. } \\
{[\mathrm{m}]}\end{array}$ & $\begin{array}{c}\text { dV } \\
{[\mathrm{km} / \mathrm{s}]}\end{array}$ & $\begin{array}{c}\text { JSpOC } \\
\text { warning }\end{array}$ & Maneuver \\
\hline $2009 / 08 / 24$ & TSX & Fengyun 1C debris & 0.11 & $605(799)$ & $191(19)$ & 13.9 & $\mathrm{x}$ & yes \\
\hline $2009 / 09 / 16$ & TSX & Cosmos 252 debris & 0.19 & $946(346)$ & 549 & 13.9 & $\mathrm{x}$ & no \\
\hline $2009 / 11 / 27$ & TSX & Cosmos 2251 debris & 0.20 & 360 & 81 & 15.2 & & yes \\
\hline $2010 / 03 / 13$ & TSX & PSLV debris & 0.14 & 2059 & 131 & 14.6 & & no \\
\hline $2010 / 06 / 27$ & TDX & Timed debris & 0.25 & 1196 & 33 & 12.8 & & yes \\
\hline $2010 / 06 / 27$ & TDX & SL-8 R/B & 2.38 & 684 & 145 & 14.1 & & yes \\
\hline $2010 / 07 / 22$ & TSX & Delta 2 R/B & 3.21 & 1122 & 230 & 12.7 & & no \\
\hline $2010 / 07 / 25$ & TSX & CZ-4C debris & 0.05 & 142 & 63 & 1.1 & & no \\
\hline $2010 / 08 / 07$ & TSX & Pegasus debris & 0.08 & $216(77)$ & $13(76)$ & 15.1 & $\mathrm{x}$ & yes \\
\hline $2010 / 08 / 20$ & TDX & Delta 1 debris & 0.15 & $1718(1936)$ & $178(160)$ & 12.9 & $\mathrm{x}$ & no \\
\hline $2010 / 11 / 25$ & TSX & Cosmos 2251 debris & 0.08 & 120 & 119 & 15.0 & & no \\
\hline $2010 / 12 / 01$ & GR1 & EXPLORER 8 & 0.65 & $208(3609)$ & $62(75)$ & 6.1 & $\mathrm{x}$ & no \\
\hline $2010 / 12 / 12$ & TDX & Cosmos 2251 debris & 0.20 & $492(360)$ & $297(20)$ & 14.8 & $\mathrm{x}$ & no \\
\hline $2010 / 12 / 12$ & TSX & Cosmos 2251 debris & 0.20 & 202 & 19 & 14.8 & & no \\
\hline $2010 / 12 / 28$ & GR1 & Cosmos 2251 debris & 0.01 & $(443)$ & $(58)$ & 14.9 & $\mathrm{x}$ & no \\
\hline $2011 / 01 / 23$ & TSX & Fengyun 1C debris & 0.02 & 745 & 76 & 14.1 & & no \\
\hline $2011 / 01 / 23$ & TDX & Fengyun 1C debris & 0.02 & 990 & 176 & 14.1 & & no \\
\hline
\end{tabular}

* ( ) given by JSpOC

Table 10. Estimated Object Size

\begin{tabular}{|c|c|c|c|c|}
\hline & $<10 \mathrm{~cm}$ & $10 \mathrm{~cm}-1 \mathrm{~m}$ & $>1 \mathrm{~m}$ & Total \\
\hline Number & 4 & 9 & 2 & 15 \\
\hline
\end{tabular}

\section{Conclusion}

Operational close approach monitoring is performed using TLEs and warnings from JSpOC as the orbit source. Potential events are analyzed carefully, and a radar tracking campaign is planned in a critical case. Based on the latest and the most precise orbit available at the time, a collision avoidance maneuver is decided.

The Orbit prediction accuracy for the TLE orbit propagation as well as for the numerical propagation showed the strong dependency on the altitude and the solar flux, especially in the radial and along-track direction. The lower altitude and the higher solar flux resulted in a poor prediction accuracy due to the higher atmospheric influence. The accuracy was also influenced by the solar flux prediction error. The resulting RMS errors are currently used in the operational close approach 
monitoring as a covariance information at the relevant altitude and the solar flux. The radar tracking accuracy analysis showed that an orbit refinement with the same precision compared to orbits based on GPS navigation solution data could be achieved for large objects.

A close approach of TerraSAR-X against a Pegasus debris was handled based on the radar tracking results of the debris. Even for the small non-cooperative object, the orbit accuracy was clearly improved compared with JSpOC and TLE orbits.

In the past collision avoidance operation for nearly 1.5 years at GSOC, three cases lead to an avoidance maneuver for TerraSAR-X and one for TanDEM-X. Most analyzed events were caused by small debris, for which an accurate orbit information is often not available. Therefore, a radar tracking is the only effective way for an orbit refinement.

\section{Reference}

[1] Krag, H., Klinkrad, H., Flohrer, T., Fletcher E., and Bobrinsky N., "The European Space Surveillance System - Required Performance and Design Concepts", Proceedings of the 8th US/Russian Space Surveillance Workshop, Space Surveillance Detecting and Tracking Innovation, Maui, Hawaii, USA, 2010.

[2] Dunagan, E., "CSM Briefings", presentation of the CSM workshop, Darmstadt, Germany, 2010.

[3] Aida, S., Patzelt, T., Leushacke, L., Kirschner, M., and Kiehling, R., "Monitoring and Mitigation of Close Proximities in Low Earth Orbit", Proceedings of the 21st International Symposium on Space Flight Dynamics - 21st ISSFD, Toulouse, France, 2009.

[4] Aida, S. and Kirschner, M., "Collision Avoidance Operations for LEO Satellites Controlled by GSOC", Proceeding of the SpaceOps 2010 Conference - SpaceOps 2010 Conference, Huntsville, Alabama, USA, 2010.

[5] Flohrer, T., Krag, H., Klinkrad, H., Bastida Virgili, B., and Früh, C., "Improving ESA's Collision Risk Estimates by an Assessment of the TLE orbit Errors of the US SSN Catalogue", Proceedings of the 5th European Conference on Space Debris - 5th European Conference on Space Debris, Darmstadt, Germany, 2009.

[6] Kahle R. and Schlepp B., "Extending the TerraSAR-X Flight Dynamics System for TanDEMX", Proceedings of the 4th International Conference on Astrodynamics Tools and Techniques 4th ICATT, Madrid, Spain, 2010. 